\title{
L'INTRODUCTION DES LANGUES NATIONALES DANS LE SYSTEME EDUCATIF AU MALI : OBJECTIFS ET CONSEQUENCES
}

\author{
Ingse Skattum \\ Université d'Oslo
}

\section{Introduction}

Les sociétés ouest-africaines sont plurilingues à des degrés divers. La situation du Mali est relativement simple, avec une vingtaine de langues (Calvet 1992 : 215 ; Canut et Dumestre 1993 : 220) ${ }^{1}$ - la Côte d'Ivoire, par exemple, qui est un cas « moyen » de plurilinguisme selon R. Chaudenson (1989 : 109), a plus de 60 langues. Le nombre plutôt restreint de langues explique en partie que le pays peut défendre son plurilinguisme non seulement dans la Constitution de $1992^{2}$ mais aussi à un certain degré dans la pratique, notamment dans l'enseignement et les médias (Skattum 2008) ${ }^{3}$.

Si le Mali fait figure de pionnier dans l'enseignement en langues nationales $(\mathrm{LN})^{4}$ parmi les pays dits francophones, le développement semble actuellement stagner. Le Rapport général du Forum National sur l'Éducation (2008), dernier document officiel sur le sujet, part d'un constat d'échec à la fois général et pour ce qui concerne l'enseignement bilingue. Le rapport recommande par conséquent de «consolider les acquis» "avant toute généralisation massive » de l'enseignement bilingue (op. cit. : 49).

Je chercherai des explications à cet échec notamment en confrontant les textes officiels avec les critères définis dans la recherche en éducation bilingue, représentée ici par un article de Nancy Hornberger (1991). Les conséquences seront présentées selon les observations faites sur le terrain par différents chercheurs. Ces conséquences jouent un rôle non négligeable pour les attitudes envers l'enseignement bilingue et, partant, pour l'utilisation des LN à l'écrit. Puisqu'il est reconnu que l'apprentissage de la lecture et de l'écriture se fait le mieux dans la langue première (L1), ou du moins dans une langue que l'enfant comprend (cf. Brock-Utne et Skattum 2009), les conséquences sont de première importance aussi pour le taux d'alphabétisation, qui en 2010 était de 26,2\% seulement (L'état de l'Afrique 2010). Et pourtant, la Banque Mondiale publie en 2007 son livre L'éducation au Mali, avec pour sous-

\footnotetext{
${ }^{1}$ SIL (Summer Institute of Linguistics) compte 50 langues endogènes au Mali (Ethnologue 2005 : 141), différence liée à la manière de définir la frontière entre langue et dialecte.

${ }^{2}$ «Le Peuple Souverain du Mali [...] proclame sa détermination à défendre les droits de la Femme et de l'Enfant ainsi que la diversité culturelle et linguistique de la communauté nationale [...] (Préambule) [...]

Le français est la langue d'expression officielle.

La loi fixe les modalités de promotion et d'officialisation des langues nationales (article 25) ».

${ }^{3}$ Ce travail comprend aussi une présentation linguistique et sociolinguistique des langues du Mali.

${ }^{4}$ Une liste des abréviations se trouve en fin d'article.
} 
titre Diagnostic pour le renouvellement de la politique éducative en vue d'atteindre les objectifs du Millénaire, sans faire la moindre mention de la langue d'enseignement ! L'impact de la langue d'instruction est donc toujours sous-estimé - pour ne pas dire ignoré - par le plus important des bailleurs de fonds.

Ce travail s'articulera comme suit: l'évolution de l'enseignement bilingue à l'école fondamentale au Mali sera présentée en (2), suivie d'un aperçu du rôle des LN aux niveaux secondaire et supérieur en (3). Les objectifs explicites et implicites des documents officiels seront confrontés à ceux définis dans la recherche internationale en (4), avant la discussion, en (5), des conséquences. Celles-ci seront analysées à l'aide de différents travaux de recherche portant sur l'enseignement bilingue au Mali et à travers les documents résultant du Forum National sur l'Éducation en 2008.

\section{L'enseignement bilingue à l'école fondamentale}

L'évolution de l'enseignement bilingue à l'école fondamentale sera décrite en (2.1), dans ses trois phases principales : l'école expérimentale de la première génération (1979-1987), la Pédagogie Convergente (PC) (1987-2002) et le Curriculum bilingue par compétences (2002- ). La synthèse de cette évolution (2.2) comprend un tableau récapitulatif ainsi qu'un tableau sur l'état actuel de cet enseignement.

\subsection{Les trois phases}

\subsubsection{L'école expérimentale de la première génération (1979-1987)}

Malgré les déclarations en faveur des LN dans la première réforme scolaire du Mali en 1962 (M. L. Traoré 2009 : 158), elles ne sont introduites à l'école qu'en 1979, près de vingt ans après l'indépendance en 1960. Quatre écoles ${ }^{5}$ abritent alors une expérimentation où le bambara (bamanankan), langue majoritaire du pays, sert de moyen d'instruction à côté du français, de la $1^{\text {ère }}$ à la $4^{\text {ème }}$ année (ibid., Hutchison et al. $1990: 11$ ). Le bambara s'est ensuite étendu à environ 80 écoles, et trois autres langues, le fulfulde (peul), le songhay et le tamasheq, majoritaires dans les régions du Macina (fulfulde) et du Nord (songhay et tamasheq), ont été introduites à une échelle modeste ${ }^{6}$. Au début, cet enseignement, appelé expérimentation de la première génération, a connu du succès, avec moins de redoublements et d'abandons et de meilleurs résultats scolaires que dans les écoles monolingues en français, dites classiques (Calvet $1988: 15-16$; M. L. Traoré 2009 : 158). Le manque de suivi a cependant fait baisser les résultats (Calvet, 1988 : 22-24; Hutchison et al. 1990 : 85). Par exemple, de 1985 à 1988, le taux d'entrée en classe de $7^{\mathrm{e}}$ année passe, pour les écoles expérimentales, de $47,66 \%$ à $29,96 \%$ (Calvet 1988 : 17). L'expérimentation de la première génération n'a plus le support des autorités : on se contente de la centaine d'écoles bilingues déjà créées, et les maîtres ne reçoivent plus aucune formation en LN (Skattum 1997).

\subsubsection{La Pédagogie Convergente (1987-2002)}

Abandonnant la première expérimentation, les autorités se tournent vers $1 \mathrm{la} \mathrm{PC}^{7}$, introduite en 1987 dans deux écoles en zone bambarophone. Après une période d'expérimentation (1987-1993) (M. L. Traoré 2009 : 158) - d'où le nom d' «expérimentation de la deuxième

\footnotetext{
${ }^{5}$ Kossa et N'djifina dans la région de Koulikoro près de Bamako, Banakoroni et Zanabougou dans la région de Ségou (Calvet $1988: 2$ ).

${ }^{6}$ Les chiffres varient selon les sources. Selon Calvet (1988:4), il y avait, en 1986-87, 78 écoles expérimentales, réparties entre 61 écoles en bambara, 6 en fulfulde, 6 en songhay et 5 en tamasheq. Selon Hutchison et al. (1990 : 6), en 1989-90, l'expérimentation comprenait 104 écoles : 83 en bambara, 6 en fulfulde, 6 en songhay et 9 en tamasheq. Selon M. L. Traoré (2009: 158), 108 écoles enseignaient en français / LN en 1987. Enfin, selon le Ministère de l'Education de Base (1994b : 5, 9), en 1992-93, on comptait 502 classes dans 109 écoles, - ce qui constitue environ 5\% des effectifs du premier cycle fondamental. alors que selon la Commission Nationale (1995 : 24), il y avait, en cette année scolaire, 108 écoles parmi les 1653 écoles du premier cycle. Voir le tableau 1 ci-dessous.

${ }^{7}$ D'abord sous le nom de Méthodologie Convergente.
} 
génération » - , on décida en 1994 de généraliser la PC. Le nom réfère à la convergence du français et d'une langue nationale comme moyens d'instruction, ainsi qu'à celle des démarches pédagogiques dans les deux langues. La PC prône, de plus, l'approche globale de la lecture ${ }^{8}$ et une pédagogie active. Elle fut d'abord introduite à la NEF (Nouvelle École Fondamentale), abandonnée après un an pour différentes raisons (Canvin 2007 : 172). Une refondation du système éducatif fut entreprise presque aussitôt et aboutit, en 1998, au PRODEC (Programme Décennal de Développement de l'Éducation). Inspiré de la NEF dans la partie enseignement fondamental, le PRODEC retint la PC comme socle méthodologique. En 2002, cette pédagogie était utilisée dans 2050 écoles à travers 11 LN (M. L.Traoré 2009 : 159).

\subsubsection{Le Curriculum bilingue par compétences (2002-)}

La PC a permis d'élaborer un « curriculum bilingue par compétences ». Adopté par la loi d'orientation sur l'éducation en 1999 (Loi n 99 / 104), ce Curriculum fut mis à l'essai en 2002 dans 80 écoles pour les deux premières années de l'école fondamentale. Le Curriculum bilingue avait «comme objectif de capitaliser les acquis de la Pédagogie Convergente et de corriger les faiblesses constatées dans son application» (Traoré 2009 : 159). Selon Y. Haïdara (2003 : 7), le Curriculum " utilise les mêmes méthodes pédagogiques, le même matériel didactique que la $P C »$. Si les statistiques officielles font la différence entre les deux types (voir le tableau 2 ci-dessous), il n'est donc pas aisé de les distinguer du point de vue pédagogique.

Selon M. L. Traoré (2009 : 159), le Curriculum était, en 2005-06, introduit dans 2550 écoles, dont les 2050 écoles à PC plus 500 écoles classiques à travers le pays. Les 2550 écoles bilingues constituaient alors 31,62\% des 8063 écoles du premier cycle. Tous les Centres d'Animation Pédagogique (CAP), cellules locales des Académies d'Enseignement (qui correspondent aux directions régionales du système éducatif national), comptaient ainsi des écoles bilingues, et $11 \mathrm{LN}$ étaient concernées.

\subsection{Remarques de synthèse}

Terminons cette présentation par quelques commentaires conclusifs sur les types d'écoles, le choix des langues, le rythme de leur introduction et le nombre d'enfants scolarisés dans les écoles bilingues.

Selon la pédagogie pratiquée, on compte six types d'écoles fondamentales au Mali depuis l'indépendance, dont un monolingue et cinq bilingues :

1. Écoles classiques (monolingues en français)

2. Écoles expérimentales de la première génération (bilingues français / LN)

3. Écoles à Pédagogie Convergente (PC) (bilingues français / LN)

4. Écoles à Curriculum bilingue par compétences (bilingues français / LN)

5. Médersas (bilingues français / arabe)

6. Écoles franco-arabes (bilingues français / arabe)

Les écoles bilingues arabe-français ne seront pas prises en compte dans cet article. On peut se référer à Bouwman (2005) pour une présentation détaillée de l'enseignement en arabe et à

\footnotetext{
${ }^{8}$ Il s'agit d'une méthode d'apprentissage de la lecture qui veut que l'élève déchiffre des mots, voire des phrases, en tant qu'image visuelle indivisible, méthode comparable à l'apprentissage de la lecture en chinois. Elle s'oppose à la méthode syllabique, qui fait le lien entre les sons de la langue et l'assemblage de syllabes. La méthode globale pose le problème du mot inconnu, le lecteur devant alors avoir recours à la méthode syllabique. Elle s'inscrit dans la méthodologie Structuro-globale audio-visuelle (SGAV) à la mode en France dans les années 1960 et 70.
} 
Tamari (2009) pour une analyse de l'utilisation (informelle) des LN dans les médersas (madrasa) au Mali.

Le choix des LN dépend de plusieurs facteurs. Pour l'expérimentation de la première génération, le choix des langues bambara, fulfulde, songhay et tamasheq fut d'ordre pragmatique, selon les autorités : "Ces quatre langues ont été choisies en raison de leur niveau d'instrumentation à la période du démarrage de l'expérimentation » (Commission Nationale 1995 : 27). Or, à l'origine de cette instrumentation (de qualité inégale pour les quatre langues) s'est trouvée une décision politique : celle faite en 1967 de les doter d'un alphabet et de leur conférer le statut de LN. La sélection de ces quatre langues au détriment de la quinzaine d'autres langues sur le terrain s'est à son tour faite en fonction du nombre de locuteurs, notamment en ce qui concerne le bambara (parlé par environ $80 \%$ de la population comme L1 ou L2) et le fulfulde (la plus importante des langues minoritaires), mais aussi en fonction de la véhicularité des langues (fulfulde au centre et songhay au nord) et de leur rôle politique et social (le tamasheq compte moins de locuteurs que par exemple le soninké). L'ordre d'introduction à l'école des autres langues nous informe également sur leur poids démographique et sociopolitique (Skattum 2008).

Au sein de la PC, de 1994 jusqu'en 2006, cet ordre est le suivant : bambara, fulfulde et songhay (introduites en 1994), dogon, soninké et tamasheq (1995), bomu (bobo) et sénoufo (syènara) (1997), bozo et minyanka (mamara) (2000), khassonké (2001). (Haïdara 2003 : 5). Deux des treize langues nationales attendent encore cette consécration : le malinké et le hassaniya (maure), la première probablement parce qu'il est très proche du bambara et le second sans doute parce que c'est une variété arabe et que l'arabe n'est pas considéré comme une langue nationale.

L'expansion de l'enseignement bilingue concerne aussi le nombre d'enfants scolarisés. Cette évolution se lit dans le tableau 1 (page suivante).

Les statistiques disponibles se prêtent assez mal à la comparaison (elles donnent le nombre d'écoles, de classes ou d'élèves, avec ou sans pourcentage du nombre total, ou encore le TBS ${ }^{9}$; néanmoins, le tableau 1 ci-dessous montre que l'enseignement bilingue au Mali se développe jusqu'en 2005-06 pour ensuite diminuer. On voit ainsi que pour la première génération, on passe de 4 écoles bilingues en 1979 à 108 en 1992, et que pour la seconde phase, qui comprend la $\mathrm{PC}$ et le Curriculum bilingue, le nombre va de 2 écoles en 1987 à 2550 (31,62\%) en 2005, pour ensuite descendre à $2338(21,1 \%)$ en 2008.

Le tableau 1 indique aussi les années marquant un changement: en 1991, les autorités recommandent d'arrêter la première expérimentation; en 1994, on décide de généraliser la PC et donc de commencer la deuxième expérimentation; en 2002 on met à l'essai le Curriculum bilingue par compétences, et en 2008, on recommande d'arrêter l'expansion de la PC.

Le passage de l'un à l'autre type est toutefois graduel et différents types d'écoles coexistent sur l'échiquier national. Dans la région de Ségou, par exemple, il y avait, lors des enquêtes d'une équipe norvégo-malienne ${ }^{10}$ en 1997, parmi les 489 écoles, 281 écoles classiques, 82 écoles communautaires (financées par la communauté, qui décide du médium d'instruction - le plus souvent, le français), 71 médersas (madrasas), 38 écoles à PC et 17 écoles expérimentales de la première génération (Direction Régionale de l'Éducation de Ségou, Inspection de Ségou I, communication personnelle).

\footnotetext{
${ }^{9}$ TBS = taux brut de scolarisation : «Les effectifs scolarisés par rapport aux effectifs scolarisables pour chaque niveau d'enseignement, calculé sur l'âge (7-12 ans; 13-15 ans; 16-18 ans)» (Banque Mondiale $2007: 26)$.

10 Financée (1996-2006) par NUFU (Programme norvégien de recherche et de formation pour le développement), dans le cadre d'une coopération de recherche entre l'Université d'Oslo, Le Centre National de la Recherche Scientifique et Technologique (CNRST) du Mali, l'Université de Bamako et L'Institut National des Langues et Civilisations Orientales (INALCO), Paris.
} 
Tableau 1 : Nombre d'écoles bilingues par rapport au nombre total d'écoles 19602008/09

\begin{tabular}{|c|c|c|c|c|c|c|c|}
\hline $\begin{array}{l}\text { Année } \\
\text { d'intro- } \\
\text { duction }\end{array}$ & $\begin{array}{l}\text { Type } \\
\text { d'école }\end{array}$ & \begin{tabular}{|l|} 
Nombre \\
d'écoles \\
/ \% \\
\end{tabular} & $\begin{array}{l}\text { Nombre } \\
\text { classes } \\
/ \%\end{array}$ & $\begin{array}{l}\text { Nombre } \\
\text { d'élèves } \\
/ \%\end{array}$ & FR & $\overline{A R}$ & $\mathbf{L N}$ \\
\hline 1960 & Classique & & & & $\mathbf{x}$ & & \\
\hline ? & $\begin{array}{l}\text { Médersas, } \\
\text { Franco- } \\
\text { arabes }^{11}\end{array}$ & & & & $\mathbf{x}$ & $\mathbf{x}$ & \\
\hline 1979 & $1^{\mathrm{e}}$ génér. & $4^{12}$ & & & $\mathbf{x}$ & & $\mathrm{BA}$ \\
\hline 1987 & PC & $2^{13}$ & & & $\mathbf{x}$ & & $\mathrm{BA}$ \\
\hline 1986-87 & $1^{\mathrm{e}}$ génér. & $78^{14}$ & & & $\mathbf{x}$ & & $\begin{array}{l}\text { BA (61) FU (6), SOG } \\
\text { (6), TA (5) }\end{array}$ \\
\hline $1989-90$ & $1^{\mathrm{e}}$ génér. & $104^{15}$ & & & $\mathbf{x}$ & $\mathbf{x}$ & $\begin{array}{l}\text { BA (83) FU (6) SOG } \\
(6) \text {, TA (9) }\end{array}$ \\
\hline 1991 & $\begin{array}{l}l^{\mathrm{e}} \text { génér. } \\
\text { gelée }\end{array}$ & & & & - & - & - \\
\hline $1992-93$ & & \begin{tabular}{|l|}
$108 /$ \\
$1653^{16}$ \\
$5 \%$ \\
\end{tabular} & 502 & 21407 & $\mathbf{x}$ & & $\begin{array}{l}\text { BA (88) FU (6) SOG } \\
\text { (6) TA (8) }\end{array}$ \\
\hline 1994 & $\begin{array}{l}\text { NEF, PC } \\
\text { généralisée }\end{array}$ & & & & $\mathbf{x}$ & & $\mathrm{BA}, \mathrm{FU}, \mathrm{SOG}$ \\
\hline 1998 & $\begin{array}{l}\text { PRODEC } \\
\text { PC } \\
\text { continuée }\end{array}$ & $\begin{array}{l}309^{17} \\
16 \%\end{array}$ & & & $\mathbf{x}$ & & $\begin{array}{l}\text { BA, BOM, DO, FU, } \\
\text { SE, SOG, SOK, TA }\end{array}$ \\
\hline 2002 & $\begin{array}{l}\text { Curriculum } \\
\text { bilingue }\end{array}$ & & & & $\mathbf{x}$ & & $\begin{array}{l}\text { BA, BOM, BOZ, } \\
\text { DO, FU, KHA, MI, } \\
\text { SE, SOG, SOK, TA }\end{array}$ \\
\hline $2004-05$ & $\begin{array}{l}\text { Statistiques } \\
\text { globales }\end{array}$ & & & $\begin{array}{l}1505903 \\
(\mathrm{TBS})^{18}\end{array}$ & & & \\
\hline $2005-06$ & $\begin{array}{l}\text { Curriculum } \\
\text { bilingue }+ \\
\text { PC }\end{array}$ & $\begin{array}{l}2550 / \\
8063^{19} \\
31,62 \%\end{array}$ & & & & & $\begin{array}{l}\text { BA, BOM, BOZ, } \\
\text { DO, FU, KHA, MI, } \\
\text { SE, SOG, SOK, TA }\end{array}$ \\
\hline 2008 & PC gelée ${ }^{20}$ & & & & - & - & - \\
\hline $2008-09$ & $\begin{array}{l}\text { Curriculum } \\
\text { bilingue }+ \\
\text { PC }\end{array}$ & \begin{tabular}{|l|}
$2338 /$ \\
$10193^{21}$ \\
$21,1 \%$.
\end{tabular} & & & $\mathbf{x}$ & & $\begin{array}{l}\text { BA, BOM, BOZ, } \\
\text { DO, FU, KHA, MI, } \\
\text { SE, SOG, SOK, TA }\end{array}$ \\
\hline
\end{tabular}

LN (Langues nationales) : $\mathrm{AR}=$ arabe, $\mathrm{BA}=$ bambara, $\mathrm{BOM}=$ bomu (bobo), $\mathrm{BOZ}=$ bozo, $\mathrm{DO}=$ dogon, $\mathrm{FR}=$ français, $\mathrm{FU}=$ fulfulde, $\mathrm{KHA}=$ Khassonké, $\mathrm{MI}=$ minyanka, $\mathrm{SE}=$ sénoufo, $\mathrm{SOG}=$ songhay, $\mathrm{SOK}=$ soninké, $\mathrm{TA}=$ tamasheq.

\footnotetext{
${ }^{11}$ Cf. Bouwman (2005), Tamari (2009).

12 Calvet (1988: 2).

${ }^{13}$ M. L. Haïdara (2005: 111).

${ }^{14}$ Calvet (1988: 4).

${ }^{15}$ Hutchison et al. $(1990: 6)$.

${ }^{16}$ Commission Nationale (1995 : 24), Ministère de l'Education de Base (1994b : 5, 9).

${ }^{17}$ Diarra et Y. Haïdara (1999).

${ }^{18}$ Banque Mondiale $(2007: 21)$.

${ }^{19}$ M. L. Traoré (2009: 159).

${ }^{20}$ Forum National sur l'Education (2008a : 49).

${ }^{21}$ Cellule de Planification et de Statistiques (2009). Ce chiffre comprend 1684 écoles à Curriculum et 654 écoles à $\mathrm{PC}(c f$. le tableau 2 ci-dessous).
} 
Le tableau 2 montre la répartition des types d'écoles en 2008-09:

Tableau 2 : Fréquence des écoles du $1^{\text {er }}$ cycle fondamental du Mali par type pédagogique 2008-09

\begin{tabular}{|c|c|c|c|c|}
\hline Type pédagogique & Fréquence & Pour cent & $\begin{array}{c}\text { Pourcentage } \\
\text { valide }\end{array}$ & $\begin{array}{l}\text { Pourcentage } \\
\text { cumulé }\end{array}$ \\
\hline Classique & 7389 & 72,5 & 72,5 & 72,5 \\
\hline Curriculum & 1684 & 16,5 & 16,5 & 89,0 \\
\hline indéterminé & 466 & 4,6 & 4,6 & 93,6 \\
\hline $\mathrm{PC}$ & 654 & 6,4 & 6,4 & 100,0 \\
\hline Total & 10193 & $100 \%$ & $100 \%$ & \\
\hline
\end{tabular}

Source : Cellule de planification et de statistiques (CPS) ${ }^{23}$.

On voit que ces statistiques distinguent écoles à curriculum bilingue (16,5\%) et écoles à PC (6,4\%). Ensemble, ces deux types d'écoles bilingues constituent $21,1 \%$ de l'ensemble. C'est toujours l'école classique monolingue en français qui domine $(72,5 \%)$. Notons cependant que «les medersas sont classées dans la même catégorie, étant des écoles qui ont le programme classique» (op.cit.). Ces écoles islamiques modernes enseignent en langue arabe et à des degrés variables en français, tout en s'appuyant, de manière informelle, sur la traduction de l'arabe en LN. Ils peuvent choisir de suivre le programme des écoles classiques et passer les examens en français (Tamari 2009). Comme ils constituent environ 20\% de l'ensemble du système formel (op. cit. : 166), l'enseignement monolingue en français paraît plus important qu'il ne l'est en réalité. Cela ne touche toutefois pas à la part relative des LN.

\section{Les langues nationales aux niveaux secondaire et supérieur}

Si on se tourne maintenant vers les niveaux secondaire et supérieur, on constate qu'après le premier cycle, les LN ne servent plus de langue d'instruction. Elles ne disparaissent toutefois pas totalement comme sujet d'étude. Comme point de départ, prenons l'aperçu de la Banque mondiale (2007) sur le nombre d'enfants scolarisés en 2004-05 et les TBS en 2003-04, aux différents niveaux :

Tableau 3 : Nombre total d'enfants scolarisés en 2004-05 et TBS en 2003-04

\begin{tabular}{|l|l|l|l|l|l|l|}
\hline & $\begin{array}{l}\text { École } \\
\text { fondam. } \\
\mathbf{1}^{\text {er }} \text { cycle }\end{array}$ & $\begin{array}{l}\text { École } \\
\text { fondam. 2 } \\
\text { cycle }\end{array}$ & $\begin{array}{l}\text { École } \\
\text { secondaire } \\
\text { générale }\end{array}$ & $\begin{array}{l}\text { École } \\
\text { secondaire } \\
\text { technique }\end{array}$ & $\begin{array}{l}\text { École } \\
\text { normale }\end{array}$ & $\begin{array}{l}\text { Enseignement } \\
\text { supérieur }\end{array}$ \\
\hline $\begin{array}{l}\text { Nombre } \\
\mathbf{2 0 0 4 - 0 5}\end{array}$ & 1505903 & 317009 & 70511 & 39669 & 9428 & 32609 \\
\hline $\begin{array}{l}\mathbf{\%} \\
\mathbf{2 0 0 3 - 0 4}\end{array}$ & $69 \%$ & $35 \%$ & $10 \% *$ & & & $286 / 100.000^{* *}$ \\
\hline
\end{tabular}

Source : Banque mondiale $(2007$ : 21, 26). Les chiffres sont extraits de deux tableaux : II.1 sur le nombre d'enfants et tableau II.3 sur les TBS.

*Cumule l'enseignement secondaire général, le technique et professionnel et l'enseignement normal, le tout rapporté à la population des 16-18 ans.

** Calculé sur 100000 habitants, l'âge variant à ce niveau.

\footnotetext{
${ }^{23}$ Je remercie Youssouf Haïdara, Direction nationale de l'Éducation de base, de m'avoir communiqué ce tableau en février 2010.
} 
Au second cycle de l'école fondamentale $\left(7^{\text {ème }}-9^{\text {ème }}\right.$ années) et dans les écoles secondaires techniques et professionnelles $\left(10^{\text {ème }}-12^{\text {ème }}\right.$ années), les LN disparaissent comme moyen et comme sujet d'enseignement. Le plus étonnant, vu l'introduction des LN à l'école fondamentale, est qu'elles soient absentes des écoles normales (instituts de formation des enseignants). Le manque de formation initiale est critiqué entre autres en 1988 par L.-J. Calvet, critique reprise par S. Traoré dans son aperçu historique de la formation des maîtres en 2000, mentionnée aussi par le ministre de l'Éducation en 2006 (M. L. Traoré 2009) et réitérée enfin dans le Rapport du Forum National sur l'Éducation en 2008 ( $c f .5$ ci-dessous). Pour toute formation, on se contente de cours d'été de 2 ou 3 semaines où la transcription ${ }^{24}$ est enseignée à des maîtres formés pour enseigner en français. Ni la structure des langues ni la didactique des langues maternelles ne sont prises en compte.

Cependant, les LN refont modestement face à l'école secondaire générale $\left(10^{\text {ème }}-12^{\text {ème }}\right.$ années), où on enseigne la transcription du bambara en filière LL (Langue, Littérature) en $11^{\text {ème }}$ année et en terminale ${ }^{25}$. La percée la plus importante s'est faite dans l'enseignement supérieur, à la Faculté de Lettres, Langues, Arts et Sciences Humaines (FLASH), où une unité de valeur obligatoire est introduite pour les étudiants de toutes les filières, portant sur la transcription des L1, à hauteur de $2 \mathrm{~h} /$ semaine. Il s'agit de 11 langues, les mêmes qu'à l'école fondamentale. Avec la création d'un Département de linguistique en 2008, on a introduit un enseignement des LN en $2^{\text {ème }}$ année aussi : d'une part, une initiation obligatoire à la transcription des LN (9 langues, le bomu et le khassonké n'étant plus incluses $(2 \mathrm{~h} / \mathrm{s})$ ) et, d'autre part, l'apprentissage d'une L2 (vocabulaire courant), qui concerne 4 langues: bamanankan, fulfulde, songhay et soninké. En $3^{\text {ème }}$ année, le département propose trois options de $2 \mathrm{~h} / \mathrm{s}$ chacune : linguistique, littérature orale et LN (la dernière concerne les 4 langues introduites en $2^{\text {ème }}$ année). Le diplôme est cependant en linguistique et non dans une LN spécifique $^{26}$. Pour avoir un diplôme dans une langue endogène, les Maliens sont donc pour l'instant toujours obligés de partir en Europe ou aux États-Unis.

Examinons maintenant les objectifs invoqués par les autorités maliennes pour introduire les LN dans leur système éducatif.

\section{Objectifs}

Commençons par jeter un coup d'œil sur les objectifs définis dans la recherche internationale pour qualifier les systèmes d'éducation bilingue (4.1). Ces objectifs seront confrontés aux documents officiels dans les trois phases esquissées ci-dessus : la première génération (4.2), la PC (4.3) et le Curriculum bilingue (4.4).

\subsection{Une typologie d'éducation bilingue}

Plusieurs typologies d'éducation bilingue ont été proposées, dont certaines d'une grande complexité $^{27}$. Parmi les plus opératoires parce que simple, est celle de Nancy Hornberger (1991). Elle définit trois modèles selon les buts qu'ils se fixent : buts linguistique, culturel et/ou social.

- Le modèle de transition vise le passage de la L1 à la L2, l'assimilation à la culture majoritaire et l'insertion sociale des minorités dans la nation (les termes majoritaire et minoritaire étant employés dans le sens de dominant et dominé). L'enseignement en L1 compense temporairement les manques de connaissances en L2.

\footnotetext{
${ }^{24}$ Dans le système éducatif malien, le terme transcription désigne l'écriture des LN en caractères latins. Ce sens se distingue de celui du terme technique utilisé en linguistique ('transcription phonétique'), mais se justifie par l'ambition des alphabets créés en 1967: " un son = une lettre ». Même si ce principe ne marche pas à $100 \%$, l'écriture reste proche de la prononciation, n'ayant pas eu le temps de s'en distancier comme c'est le cas par exemple pour le français et l'anglais.

25 Information de N'do Cissé, chef du Département de linguistique, Université de Bamako, le 20.02.2010

${ }^{26}$ Je remercie N'do Cissé aussi pour toutes les informations concernant la FLASH.

${ }^{27}$ Pour plus de détails, voir par exemple Skattum (1997) et Brock-Utne et Skattum (2009).
} 
- Le modèle de maintien vise le pluralisme à travers le maintien et la protection de la L1 ainsi que la confortation de l'identité ethnique et l'affirmation des droits de l'homme.

- Le modèle d'enrichissement vise également le pluralisme culturel, mais va plus loin, soutenant non seulement le maintien mais aussi le développement et l'expansion des langues minoritaires (dominées), et l'autonomie des différents groupes culturels, intégrés dans la nation.

Cette typologie peut nous éclairer sur les objectifs tant explicites qu'implicites de l'introduction des LN comme moyens d'instruction au Mali.

\subsection{L'école expérimentale de la première génération (1979-1987)}

Selon les documents officiels, l'école expérimentale de la première génération avait avant tout un but culturel : la revalorisation et le développement de la culture et des langues de la nation :

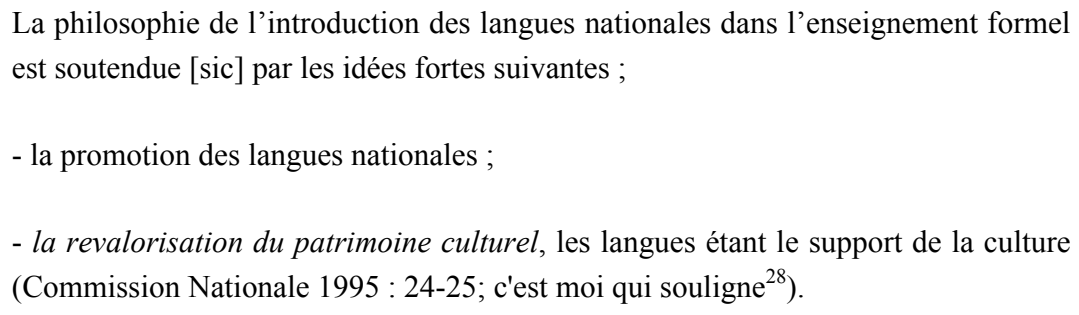

Le but social paraît également important: résoudre la déperdition scolaire qui déjà à l'époque était évidente, et qu'on imputait en partie à l'inadaptation de l'école au milieu :

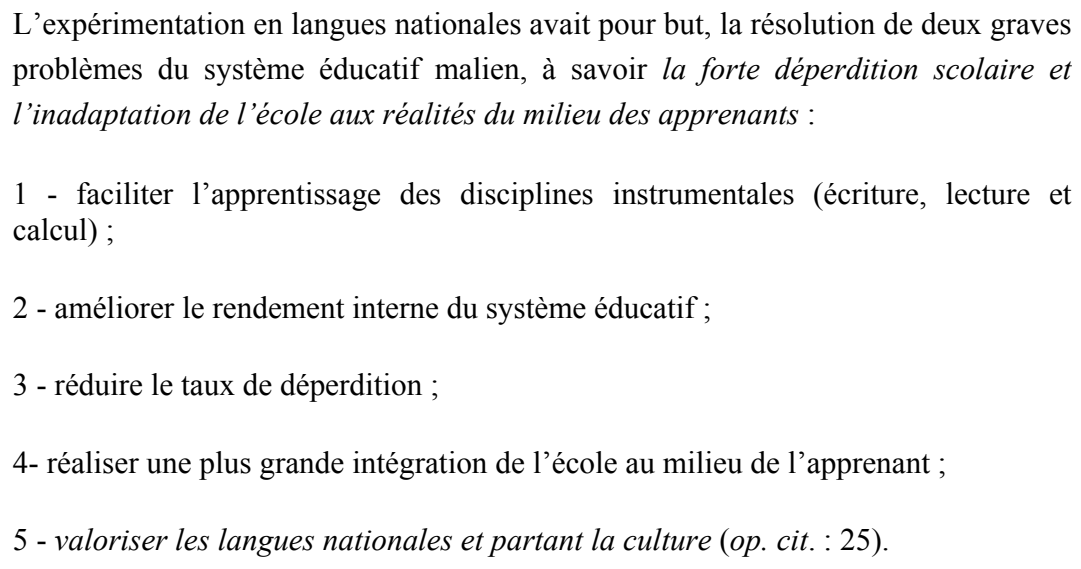

Ce but social pourrait s'inscrire dans les trois modèles : selon les convictions, on peut considérer que c'est la transition, le maintien ou l'enrichissement qui mène à l'amélioration des résultats scolaires. Cependant, la valorisation des LN est toujours mentionnée, indiquant un modèle de maintien ou d'enrichissement.

Le but linguistique n'est pas mentionné. Deux faits permettent cependant de l'inférer : 1) Les examens de fin de cycle de l'école fondamentale étaient faits uniquement en et sur le français (Ministère de l'Éducation 1994a) ; 2) Le programme des écoles classiques était transposé tel quel aux écoles bilingues (Commission Nationale 1995 : 27). Ces mesures inscrivent la réforme dans un modèle de transition.

\footnotetext{
${ }^{28}$ Désormais, c'est moi qui souligne si rien d'autre n'est indiqué.
} 
On voit que l'expérimentation de 1979 contient une contradiction entre objectifs et modèle d'éducation bilingue : le but culturel, tel qu'il s'exprime dans les documents officiels et aussi dans la Constitution ( $c f$. la note 2 ci-dessus), demanderait le maintien ou l'enrichissement, alors que le but linguistique sous-tendu implique la transition. Cette contradiction explique en partie ce qu'il faut bien nommer l'échec de la première génération d'écoles expérimentales ${ }^{29}$.

\title{
4.3. La pédagogie convergente (1987-2002)
}

Outre sa pédagogie, qui se veut plus moderne, la $\mathrm{PC}$ se distingue de la première expérimentation par le fait que l'éducation bilingue se poursuit jusqu'à la fin du premier cycle (six ans).

La PC part du principe pédagogique - qui semblerait évident - que l'enfant apprend mieux à lire et à écrire dans sa L1, et que cette maîtrise facilite l'accès à d'autres connaissances, y compris la L2. Aussi les présentations de la NEF et du PRODEC font-elles explicitement mention du but linguistique :

- NEF :

L'objectif général assigné à cette nouvelle stratégie est: Développement d'un bilinguisme fonctionnel chez l'enfant. Pour atteindre cet objectif, la pédagogie convergente part de l'idée que l'appropriation de l'écrit en langue maternelle détermine tous les apprentissages ultérieurs (Commission Nationale 1995 :34).

- PRODEC :

En choisissant la pédagogie convergente de l'enseignement des langues maternelles et du français, le Département de l'Éducation de Base s'oriente résolument vers un multilinguisme fonctionnel scolaire (PRODEC 2000 : 35).

La NEF aspire même à une maîtrise « parfaite » aussi bien de la L1 que de la L2 :

\begin{abstract}
Ainsi, face au double échec des politiques éducatives sur le plan de l'utilisation du français et celui de la valorisation des langues nationales, la pédagogie convergente constitue une bonne alternative pour recentrer la problématique de l'éducation, car elle permet [...] de [...] renover [sic] les méthodes pédagogiques dans les écoles dans le sens d'une maîtrise parfaite de la langue maternelle de l'apprenant et de la langue seconde et rapprocher ainsi l'école des populations (Commission Nationale 1995 : 33-34).
\end{abstract}

Or, les termes (non définis dans ces documents) de bilinguisme fonctionnel (NEF) ou de multilinguisme fonctionnel scolaire (PRODEC), ne désignent pas d'ordinaire un bilinguisme parfait (donc équilibré) dans les deux langues. Le bilinguisme fonctionnel reconnaît au contraire des fonctions différentes aux langues qui se trouvent en situation de diglossie. Au Mali, c'est le français qui fonctionne comme langue de prestige, la langue "haute»: officielle, formelle, écrite, etc., alors que les LN assument le rôle de langues « basses »: communication familiale, informelle, orale ${ }^{30}$. Le but linguistique (obtenir un bilinguisme fonctionnel) inscrit donc la PC aussi dans un modèle de transition, plutôt qu'un modèle de maintien ou d'enrichissement.

\footnotetext{
${ }^{29}$ Commission Nationale (1995 : 30-32) : «Les Difficultés rencontrées dans les écoles expérimentales 1ère génération »; $c f$. aussi Tréfault (1999: 50).

${ }^{30}$ En réalité, il s'agit de plusieurs diglossies « enchâssées » (Calvet 1987) car le bambara, majoritaire et dominant parmi les langues nationales, détient un rôle intermédiaire entre le français et celles-ci (Dumestre 1994). À leur tour, certaines langues dominantes dans leurs régions fonctionnent comme des langues hautes vis-à-vis des langues locales.
} 
Pour le « père » de la PC, M. Wambach, les buts linguistique et culturel ne sont cependant que des moyens pour atteindre un but social, à savoir le développement de l'individu :

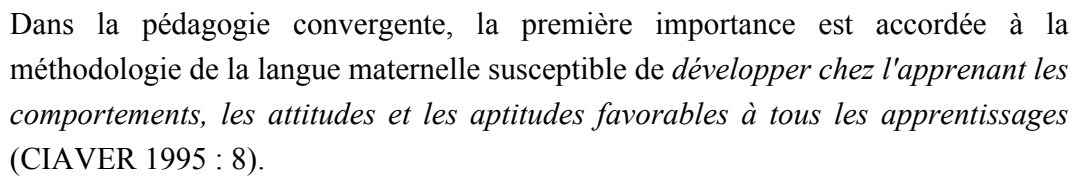

Pour la communauté internationale ainsi que les autorités maliennes, c'est un autre but social qui, depuis une quinzaine d'années, fraye son chemin: le développement de la collectivité par la littératie :

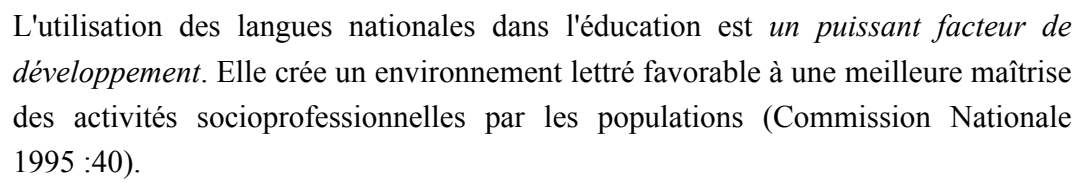

On peut objecter que si l'école est, en effet, un puissant vecteur de développement, elle ne peut à elle seule créer un environnement lettré ; pour cela, il faudrait un aménagement linguistique du pays qui permette aux Maliens de s'en servir dans leur vie quotidienne et la sphère publique. Comme le constate M. L. Traoré (2009 : 156), il faudrait « [avoir] à l'esprit qu'il serait important de parler de leur utilisation dans d'autres sphères de la vie telles que la justice, la communication, l'administration publique, etc. ».

\subsection{Le Curriculum bilingue par compétences (2000- )}

Il m'a été impossible de trouver dans les documents officiels une distinction en termes pédagogiques entre la $\mathrm{PC}$ et le Curriculum bilingue par compétences (voir 2.1.3 ci-dessus). Selon R. Chaudenson (2006 : 107), se référant à B. Maurer (2004), « des modifications ont été introduites en matière de didactique des langues; elles prolongent la PC en la modifiant. Sur le plan des horaires, ces changements augmentent globalement la place faite au français ». Selon les documents maliens auxquels j'ai eu accès, la différence semble résider surtout dans les efforts pour fournir les écoles bilingues en matériel didactique en LN. Selon Y. Haïdara (2003 : 6), l'insuffisance de matériel didactique en LN constituait en effet le point le plus faible de la PC, et L. Dembélé (2006) constate que "[l]e manque de manuel[s] est devenu si aigu qu'il est considéré actuellement comme l'obstacle majeur aux progrès de l'école primaire en général et en particulier à la bonne marche des innovations. Des écoles de la première génération au curriculum en passant par la pédagogie convergente le constat est le même» (op. cit. : 1). Un aperçu des rares documents disponibles en LN pour la première génération est suivi d'une description des ateliers annuels d'élaboration de tels documents, conçus pour la PC et poursuivis pour le Curriculum. L'état de production en 2004 était de 98 titres répartis sur les 11 langues, avec 66 titres en langue et sciences de l'éveil (histoire, géographie) et 32 en mathématiques. À partir de 2004, les ateliers focalisent sur la production de « documents d'accompagnement du curriculum» (op. cit. : 5), avec un bilan de 28 titres en 2006, répartis non seulement sur les $11 \mathrm{LN}$ mais aussi sur le français. La continuité mais aussi la différence entre la production scolaire pour les écoles à $\mathrm{PC}$ et celles à Curriculum sont soulignées par Dembélé (op. cit. : 5). Il s'agit en effet non seulement d'augmenter la quantité mais aussi de combler des lacunes :

Ces ateliers sont la suite logique de ceux de la pédagogie convergente. Ils se tiennent au même lieu [Niono] et à la même période. L'organisation du travail est la même qu'en pédagogie convergente. La différence réside fondamentalement au niveau des termes de référence. Lors de ces ateliers sont surtout traités les contenus $\mathrm{du}$ programme de formation du curriculum qui ne sont pas pris en compte par la pédagogie convergente. Cela concerne les cinq (5) domaines: langues et 
communication (LC), Mathématiques Sciences et Technologie (SMT), Sciences

Humaines (SH), Arts, Développement de la personne (DP).

Dembélé s'inquiète cependant pour le destin des langues minoritaires :

[S]i l'adoption de la politique du livre a été salutaire, son application pose problème. Pour les langues nationales, seules les langues majoritaires intéressent les éditeurs. Les langues dites minoritaires risquent d'en être les parents pauvres. À ce rythme beaucoup de nos langues nationales risquent de rester en marge des innovations car ne disposant d'aucun manuel pouvant répondre aux besoins pédagogiques en vigueur (op. cit. : 8).

L'égalité de principe de toutes les LN ( $c f$. la Constitution de 1992) rencontre ainsi la réalité du terrain : c'est la loi du plus fort qui prévaut dans la « guerre des langues » (Calvet 1987).

\section{Conséquences}

Les conséquences de l'introduction de la PC et du Curriculum bilingue seront maintenant examinées à la lumière d'un certain nombre de travaux de recherches : d'abord ceux de l'équipe NUFU (composée de chercheurs maliens, norvégiens et français) ${ }^{31}$, réunis dans deux numéros du Nordic Journal of African Studies, 6-2 (1997) et 9-3 (2000) (www.njas.helsinki.fi/) (5.1), ensuite ceux de chercheurs de différents horizons (5.2), et enfin le Rapport et la Déclaration du Forum National sur l'Éducation (2008) (5.3).

\subsection{Les travaux de l'équipe NUFU}

Notamment trois aspects sont soulevés par l'équipe NUFU: la maîtrise par les élèves de la L1 à l'écrit (5.1.1); le transfert de leurs compétences de L1 à L2 (5.1.2) et les attitudes des maîtres et des parents d'élèves envers l'enseignement en LN (5.1.3).

\subsubsection{La maîtrise de l'écrit en langues nationales}

L'un des constats faits sur le terrain par notre équipe est le faible niveau des compétences des élèves à l'écrit, tant en bambara (Skattum 2000) qu'en français (Skattum 2004). Même dans leur L1, les textes produits sont difficiles à lire, parfois illisibles.

Ce constat se base sur l'analyse des récits faits par 28 élèves en $5^{\mathrm{e}}$ année. En bambara, c'est surtout la segmentation des mots qui pose problème. Les mots sont tantôt agglutinés, tantôt séparés à tort. Ces erreurs viennent d'une méconnaissance de la structure de la langue, méconnaissance souvent partagée par les maîtres, comme le montrent des observations en classe (Doumbia 2000). On remarque aussi la transcription souvent impressionniste des sons et le manque de ponctuation et de lettres majuscules pour délimiter les phrases.

Les raisons de cette orthographe défaillante sont nombreuses. Le manque de matériel didactique en est la première. L'école fondamentale dispose de moins de textes écrits en LN qu'en français et surtout, ne dispose pas de grammaires pédagogiques dans les LN, ni de guides pour les maîtres, ni de manuels pour les enfants (Kanè 2000). C'est pourquoi notre équipe a décidé d'en faire. Nous avons d'abord travaillé sur la terminologie grammaticale, faisant état des travaux existant, les harmonisant et les complétant, avant d'élaborer deux prégrammaires, des 'Éveils à la langue' : une en bambara (Bamanankan maben nyebilagafe) et une autre en fulfulde (Naatirde naahu fulfulde), destinées aux maîtres des première et deuxième années. La pré-grammaire bambara a ensuite été incorporée à une grammaire pour

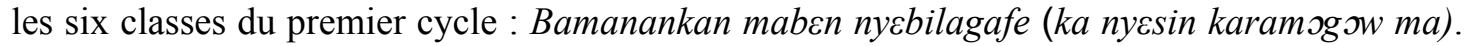
Après avoir été testées dans les classes et soumises à l'appréciation des chercheurs de toutes les institutions maliennes travaillant sur les LN, la pré-grammaire fulfulde et la grammaire bambara ont été publiées à Bamako en 2006. La même année, elles ont été reconnues par le

\footnotetext{
${ }^{31}$ See project presentation : http://www.hf.uio.no/ikos/english/research/projects/mali/index.html
} 
Ministre de l'Éducation pour «utilisation [...] dans les écoles fondamentales, dans les établissements de Formation des Maîtres et les structures de l'éducation non formelle ». Notre espoir est que ces grammaires scolaires pourront servir à un enseignement systématique de la langue nationale, à l'instar de celui dont bénéficie le français.

La deuxième raison de la défaillance est, précisément, le déséquilibre qui existe entre les enseignements de L1 et L2. Alors que la grammaire française est enseignée de manière systématique, la langue bambara est enseignée à travers d'autres matières, et les fautes corrigées sans explication grammaticale (Doumbia 2000).

La troisième raison est la formation des maitres. Les LN n'ont jamais fait partie de la formation initiale des maîtres, exception faite d'une brève période entre 1992 et 1994 :

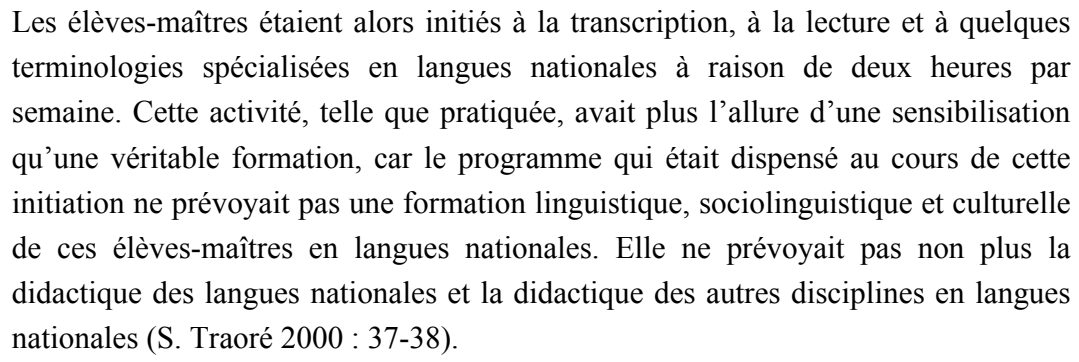

Cette façon d'enseigner les LN continue, sous la forme de stages de formation, le seul enseignement qui existe en la matière. L'aspect pédagogique (pédagogie active et en grands groupes, approche globale à la lecture) domine toujours sur l'aspect linguistique et la partie linguistique se concentre sur la transcription, pas sur la structure de la langue, ni sur la didactique de L1. Le manque de manuels en LN aggrave l'insuffisance de cette formation:

Il a été constaté, à travers les missions de suivi pédagogique et les rapports d'évaluation des stages, que la formation en langue nationale est nettement insuffisante et la documentation relative à ces langues peu accessible. À part les notes prises au cours des stages de formation, les maîtres ne disposent en fait pas de documents de référence pour approfondir et renforcer les connaissances acquises dans le domaine des langues nationales (S. Traoré $2000: 38$ ).

La quatrième raison, enfin, est l'environnement sociolinguistique au Mali. Le bambara et les autres LN demeurent des langues essentiellement orales, et les élèves ne voient pour ainsi dire pas de bambara imprimé, qui leur aurait permis d'intérioriser son orthographe.

Ces lacunes mettent en danger la prémisse première de la réforme, à savoir que l'écriture et les concepts académiques doivent être maîtrisés dans L1 avant l'apprentissage de L2. Cette prémisse, basée entre autres sur les recherches de James Cummins (1979) (Hutchison et al. 1999), est clairement intégrée dans les objectifs de la PC :

\begin{abstract}
L'introduction de la deuxième langue n'est souhaitable que lorsque les plus importants comportements, ceux concernant l'écrit notamment, sont acquis en première langue. En effet, la prise de conscience de sa propre langue se produit à travers les activités d'écrit. Une bonne appréciation de l'écrit est vraisemblablement décisive pour tous les autres apprentissages y compris ceux de la $2^{\text {ème }}$ langue (Commission Nationale 1995 : 34).
\end{abstract}

Les faibles compétences en L1 est une conséquence qui mine les buts linguistique et social de la réforme, aux niveaux individuel et collectif. Au niveau national, elle nuit aussi au but culturel. 


\subsubsection{Le transfert des connaissances en $\mathrm{L} 1$ à $\mathrm{L} 2$}

Le transfert des connaissances en L1 à L2 constitue une autre prémisse de la réforme. Or, nos observations montrent que ce transfert n'est pas bien assuré. Prenons comme exemple l'enseignement des mathématiques. Nous avons assisté au $4^{\mathrm{e}}$ stage de formation de haut niveau à la pédagogie convergente (Direction Régionale de l'Éducation de Ségou 1997). Le document de travail, intitulé Méthodologie de l'apprentissage de la langue nationale et des mathématiques (Krinic et Wambach 1997), contenait 17 exercices, tous en français, sans que référence soit faite à l'enseignement initial en bambara :

\footnotetext{
[...] il est entièrement rédigé en français, aussi bien les exercices et les objectifs que le déroulement de la leçon. Il est muet sur une exploitation éventuelle des cours dispensés par les maitres en bambara. Aucun exemple de traduction (ou d'adaptation) des exercices n'est proposé en bambara. Aucune référence n'est faite aux difficultés terminologiques et au contenu culturel de certaines notions susceptibles d'apparaître lors du passage en bambara. Le rapport que doit entretenir la langue nationale et le français dans l'enseignement du calcul n'est pas non plus abordé par le stage [...] Enfin, bien que l'enseignement des mathématiques soit au cœur des préoccupations du $4^{\mathrm{e}}$ stage [...], aucune de ses 14 recommandations ne pose explicitement les problèmes liés au transfert en français des connaissances mathématiques acquises dans la langue maternelle (Kanouté 2000 : 84).
}

Le problème ici est de savoir comment un maître de $4^{\mathrm{e}}$ ou de $5^{\mathrm{e}}$ année doit exploiter le vocabulaire mathématique que l'élève a acquis de la $1^{\mathrm{e}}$ à la $3^{\mathrm{e}}$ année dans la langue nationale et comment il doit faire le « dosage des langues ». Ce même problème se pose évidemment pour d'autres matières.

\subsubsection{Les attitudes linguistiques des maîtres et des parents}

Quelles sont les conséquences de ces lacunes (faibles compétences en L1, transition non assurée de L1 à L2) sur le choix des parents? Notre équipe a recueilli des informations sur l'attitude des maîtres et des parents vis-à-vis de l'introduction des LN à l'école.

\subsubsection{Les attitudes des maîtres}

Auprès des maîtres, nous avons mené une enquête quantitative (Haïdara 2000). On note une légère adhésion $(55,38 \%)$ à la réforme, mais il faut prendre au sérieux les résistances, qu'on peut résumer ainsi (op. cit. : 60-62) :

1. L'enseignement en langue nationale interfère négativement sur le niveau en français.

2. Le niveau d'évolution et d'aménagement des LN est insuffisant.

3. Les LN ne sont pas enseignées en dehors du Mali.

4. L'aspect expérimental de l'innovation confère à l'enseignement en LN une incertitude quant à son aboutissement. L'expérience de la Guinée a été évoquée et certains avancent que les enfants qui sont dans des classes expérimentales sont des « cobayes».

5. Le déplacement des parents défavorise un enfant qui a déjà été scolarisé dans une première langue et qui se retrouve dans une autre zone linguistique.

6. Le rôle de promotion sociale du français a en particulier été évoqué. En effet, beaucoup d'enseignants soutiennent qu'aucune politique linguistique ne définit le rôle des LN en dehors de l'école fondamentale ; mieux, dans la pratique, seul le français donne accès à un emploi administratif. Le français, en effet, de par son statut de langue officielle, acquiert «une valeur marchande » que n'ont pas les LN. Les récentes dispositions pour le recrutement dans les ONG attestent, d'ailleurs, que la capacité d'une langue à donner du travail sert cette langue plus que tout autre facteur. En effet, quand des ONG ont commencé à exiger, des candidats aux différents tests de recrutement, l'aptitude à lire et à écrire dans la langue du milieu, les salles de formation en LN n'ont pas désempli. 
L'enquête révèle par ailleurs que le facteur déterminant pour une attitude positive, bien plus que l'âge, l'expérience ou l'origine du maître, est l'information sur l'intérêt, pour l'enfant, d'apprendre dans sa langue maternelle. Il faut donc continuer la sensibilisation non seulement des maîtres, mais de la population entière.

\title{
5.1.3.2. Les attitudes des parents
}

Les attitudes des parents ont été étudiées de manière qualitative, à travers des observations et des entretiens dans un village près de Ségou (Opheim 1999, 2000). Les parents interviewés sur la question paraissent plus négatifs que les maîtres, d'abord parce qu'ils estiment qu'ils connaissent déjà le bambara :

\begin{abstract}
D'abord, quand on avait dit que c'était le bambara [lors des tournées de sensibilisation], les paysans n'appréciaient même pas, parce que pour eux, aller à l'école, c'est apprendre quelque chose, essayer d'avoir quelque chose qu'on ne connaît pas. - Mais, nous connaissons le bambara! Qu'est-ce qu'on va raconter ? (Opheim $2000:$ 155).
\end{abstract}

Mais c'est surtout la dégradation des résultats scolaires qui joue un rôle déterminant :

\begin{abstract}
Autrefois, si on avait fait trois ou quatre ans à l'école, on était capable d'écrire des lettres. Mais ça fait longtemps maintenant que les choses ne sont plus comme ça. L'école est devenue très compliquée ces derniers temps. Maintenant, on fait l'alphabétisation [en bambara] et l'école [en français] en même temps. On a constaté ceci : nos enfants ne savent plus écrire des lettres en $4^{e}$ année (AC.) (op. cit. : 156 ; c'est l'auteur qui souligne).
\end{abstract}

Comme les maîtres, les parents trouvent que l'introduction des LN influe négativement sur le niveau en français. C'est parce que le bambara occupe maintenant une partie du temps scolaire, et que les élèves doivent apprendre deux langues au lieu d'une seule, que les résultats se dégradent. Ils voient l'aggravation du chômage comme une conséquence de cette dégradation.

Ils préfèrent le français, à cause de tout ce qu'il apporte au niveau socio-économique :

Votre langue, c'est la langue de l'argent et du salaire fixe. Si quelqu'un a réussi, estce que ce n'est pas une affaire d'argent ? Si tu connais le français, tu peux voyager et connaître le monde, et personne ne peut te tromper. Avec notre langue, tu ne sais rien (N.M.) (op. cit. : 155).

C'est l'utilité du français qu'on a vu à [...], parce que nos enfants qui l'ont appris sont devenus des personnes importantes. Mais jusqu'à ce jour, nous n'avons pas encore vu que les élèves qui ont étudié le bambara en ont tiré profit. (O.C.) (ibid.).

Après deux mois d'observations, M. Opheim conclut ainsi :

Deux traits de la vie malienne aggravent les problèmes sociolinguistiques de l'enseignement bilingue : le manque d'un environnement écrit, tant en bambara qu'en français, et l'absence du français comme moyen d'expression orale. Ces traits sont plus accentués à la campagne que dans les villes, dû au taux de scolarisation plus élevé dans les villes, mais aussi à l'absence d'emplois à la campagne qui demanderaient des connaissances scolaires. Le seul emploi «intellectuel» au village est en fait celui d'enseignant. Les paysans de [...] n'ont besoin ni de connaître d'autres langues que le bambara, ni de savoir lire et écrire pour accomplir les tâches quotidiennes, car il y a toujours quelqu'un d'alphabétisé pour régler les quelques « affaires de papier » qui apparaissent. (op. cit. : 156). 
Comme le dit l'un des interviewés : "Si l'on ne devient pas fonctionnaire, pourquoi ne pas rester à la maison? »(S.M.) (op. cit. : 157 ).

Un des rares avantages apportés par la connaissance du bambara écrit serait de pouvoir « dire des choses dans une lettre que personne ne connaît sauf toi. Comme ça, tu peux garder des secrets » (op. cit. : 155). A. Mbodj-Poye en relève un autre dans sa thèse (2007), repris dans un article (Mbodj-Poye et Van den Avenne 2009: 302) : la tenue de cahiers personnels " où sont consignés événements marquants et données variées à conserver ». Ces cahiers, bilingues français-bambara, sont tenus par de jeunes adultes d'un village ayant accueilli l'une des premières écoles bilingues en 1979. La tenue des cahiers représente ainsi l'un des acquis possibles de l'enseignement bilingue.

L'utilité de l'école est évidemment de première importance pour l'adhésion de la population. Victoria J. Baker (1998), qui a fait des recherches sur le terrain dans sept pays en voie de développement, conclut que le facteur le plus important pour le choix du médium d'instruction est son utilité. Mais l'interprétation de cette utilité peut varier : vu d'en haut, ce serait l'unité nationale ou le progrès technologique, vu d'en bas, ce serait la lecture et l'écriture au quotidien et l'accès au marché de travail (op. cit. : 34).

\subsection{D'autres travaux de recherche}

Si l'équipe NUFU a montré les limites de la PC sur le terrain, B. Maurer (2007) va plus loin dans sa critique. Il montre que les principes pédagogiques de la PC sont bâties sur une version mal comprise de la méthode SGAV (voir ci-dessus note 7) ${ }^{32}$ et qu'au lieu de prendre en compte la différence entre l'apprentissage d'une L1 et d'une L2, les promoteurs de la PC ont opté "pour un parallélisme méthodologique: on enseigne la L2 comme la L1, selon les mêmes principes communicatifs (bain de langue, absence de réflexivité dans les apprentissages linguistiques, rapports interlinguistiques non pensés ») (Maurer 2010 : 175). Comme l'équipe NUFU, il montre que les horaires privilégient l'enseignement en français, et que la dimension métalinguistique dans l'apprentissage des LN fait défaut. La PC n'est pas, en fait, une didactique linguistique, mais une didactique générale.

En contraste avec la plupart des études de l'équipe NUFU (Doumbia, Kanè, M. L. Haïdara, Kanouté, Opheim, Skattum, S. Traoré, tous 2000), et avec celle de Maurer (2007), M. Canvin (2003) arrive à une appréciation positive de la PC. À l'instar de nombre d'études quantitatives, sa thèse, une étude qualitative portant sur un complexe scolaire, conclut que l'école bilingue souffre de moins de redoublements et d'abandons que l'école classique. Comme nombre d'études quantitatives aussi, celle de Canvin souligne l'importance d'autres paramètres que la langue pour la réussite scolaire (manque de matériel, d'enseignants, de salles de classes, affection des maîtres sans prendre en compte leur L1...).

G. Dumestre (1997, 2000), également membre de l'équipe NUFU, trouve comme Canvin que la langue d'instruction ne peut à elle seule expliquer la situation « catastrophique ", mais il met en avant les facteurs culturels : "les mauvais résultats du système éducatif sont liés à des tendances lourdes qui plongent dans la géographie et l'histoire du Mali, et dans l'organisation, ancienne et contemporaine, des communautés humaines qui le constituent » (Dumestre 1997 : 32-33, c'est l'auteur qui souligne). Il rappelle que la plupart des pays situés dans la frange sahélienne de l'Afrique se trouvent parmi les plus mal classés du monde en matière d'éducation, que la culture de l'écrit y est peu implantée, la solitude mal appréciée et l'obéissance requise devant les aînés, que le métier d'enseignant est dévalorisé, la hiérarchie des âges demandant une certaine distance de la part des adultes vis-à-vis des enfants et enfin que les écoles coraniques, où la mémorisation et l'obéissance tiennent lieu de méthode

\footnotetext{
${ }^{32}$ En soi mal adaptée à la réalité malienne : «La méthodologie S.G.A.V. exige la mise en place d'un dispositif lourd d'enseignement qui comporte des formations spécifiques aux enseignants par le biais de stages, des coûts matériels importants de mise en place (magnétophonel laboratoire de langue...), et des dispositifs d'enseignement contraignants: nombre réduit d'apprenants/ enseignement hebdomadaire intensif/ formation sur une longue durée (2 à 3 ans) » (AUF s.d., s. p.).
} 
pédagogique, constituent le modèle de référence de l'institution scolaire. Quant à l'introduction des LN dans le système éducatif, il trouve que "la mauvaise gestion $d u$ plurilinguisme fait de celle-ci un fardeau supplémentaire, malheureusement à forte connotation idéologique et politique » (op. cit. : 44).

Dans sa thèse (2007), T. Tréfault montre qu'il est, comme Canvin, plutôt favorable aux méthodes de la PC, et met, comme Canvin et Dumestre, en avant les facteurs externes à l'école. Étudiant la vie quotidienne de deux écoles bilingues situées dans la même zone bambarophone, il arrive à la conclusion que c'est la différence d'accueil fait à ces deux écoles (l'un des villages étant favorable, l'autre non) qui explique en grande mesure leurs résultats divergents - malgré l'environnement apparemment semblable.

Citons enfin trois thèses soutenues à l'école doctorale de l'Université de Bamako, l'Institut Supérieur de Formation et de Recherche Appliquée (ISFRA), entre 2005 et $2010^{33}$.

M. L. Haïdara (2005), comme Tréfault positif envers les méthodes de la PC, y compris la SGAV, montre néanmoins que des lacunes existent qu'il faudrait éliminer par « une politique de formation et de recrutement d'enseignants plus appropriée à la situation, d'une politique soutenue de dotation en matériel et manuel de qualité dans les différentes langues, d'un plan de communication incluant la politique de promotion des langues nationales » (op. cit. : introduction, s.p.). La sensibilisation des parents est particulièrement importante : une enquête sur le rythme des inscriptions à Ségou a montré que les écoles enseignant en LN n'ont commencé à se remplir que lorsque les écoles enseignant en français avaient «fait le plein » (op. cit. : 243-252).

C. T. Sangaré (2007) examine l'impact des facteurs linguistique et didactique dans l'enseignement de la philosophie au lycée. Les lycéens, de L1 bambara et fulfulde, sont tous passés par l'école "classique ». Les résultats d'un test de compréhension montrent que les résultats sont pour la plupart très faibles, et qu'une L2 comme langue d'instruction constitue un obstacle sérieux à la compréhension d'une discipline, même après 12 ans d'enseignement dans cette langue.

H. Naparé (2010) examine les représentations des parents d'élèves de l'école bilingue et le vécu des enseignants et des élèves de ces écoles au pays dogon. Il montre que le choix du tôrôso comme dialecte standard ${ }^{34}$ suscite des ressentiments, puisqu'il s'enseigne aussi dans des zones non tôrôsophones, par des enseignants d'autres dialectes, et que ces faits, parmi d'autres, font revenir les parents vers le français. Il montre de plus que les maitres s'estiment insuffisamment formés, qu'un grand nombre d'enseignants sont non professionnels, sans connaissance de la PC, que certaines techniques de la PC sont stigmatisées, qu'il y a un manque de manuels en langue dogon, que les suivis pédagogiques sont rares et irréguliers, et que «l'environnement lettré des apprenants est d'une pauvreté très aiguë» (op. cit. : 3). Pour ces raisons, il prévoit que l'école bilingue va régresser plutôt qu'avancer en pays dogon.

\subsection{Le Rapport général et la Déclaration du Forum National sur l'Éducation (2008)}

Les problèmes et les lacunes soulevés dans tous ces travaux sont repris par le Rapport général du Forum National de l'Éducation de 2008. Cependant, la PC, au centre des refontes antérieures de l'école fondamentale (généralisation décidée en 1994 par la NEF et confirmée en 1998 par le PRODEC), ne figure ici que sous forme d'une précision sous l'une des très

\footnotetext{
${ }^{33}$ Les auteurs ont tous bénéficié du financement de NUFU et de l'encadrement de membres de l'équipe.

${ }^{34}$ Le dogon consiste en 12 à 15 dialectes (ou même 20, selon Plungian et Tembiné 1994 : 164) sans intercompréhension sauf pour les dialectes voisins. La différence est si grande qu'on discute s'il s'agit de langues et non de dialectes. Aucune variété commune n'existe qui permettrait la communication interdialectale (ibid.). Dans ces conditions on comprend la frustration des populations du choix fait par les autorités d'un dialecte particulier comme standard. Que ce soit le tôrôso s'explique par l'influence de l'ethnologue Marcel Griaule, dont les travaux portaient sur ce groupe précis. (Pour une discussion générale de la question de standardisation et d'harmonisation des langues et dialectes en Afrique, Brock-Utne et Skattum 2009).
} 
nombreuses recommandations. Celle-ci apparaît dans le chapitre rendant compte des résultats de la "Commission Éducation de Base, Alphabétisation et Langues Nationales ». (Aucune mention n'est faite des LN dans les chapitres sur l'enseignement supérieur ou l'enseignement secondaire général, technique et professionnel.) Faisant état des réticences de la population envers cette pédagogie, le Rapport recommande ici de :

\begin{abstract}
- poursuivre la mise en œuvre de la PC (Pédagogie Convergente) et du curriculum en veillant à leur suivi, leur évaluation, à la formation du personnel chargé de l'animation et de la supervision et à la mise en place du matériel conséquent. Toutefois, afin de vaincre les réticences envers le curriculum et la pédagogie convergente, le Forum recommande de mettre en œuvre les stratégies suivantes :

$>$ consolider les acquis de la pédagogie convergente : il s'agit de capitaliser les points forts de la $\mathrm{PC}$, notamment sur le plan pédagogique et production de matériels didactiques (les ateliers de Niono);

$>$ capitaliser les résultats de la mise à l'essai ;

$>$ assurer une préparation matérielle (y compris les ressources humaines) suffisante avant toute généralisation massive;

$>$ procéder à une préparation psychologique de tous les partenaires (syndicats, APE [Association des Parents d'Élèves], CGS [Comité de Gestion Scolaire], ONG, élus) : information, sensibilisation, formation (Forum National 2008a : 49).
\end{abstract}

Parmi les points forts on mentionne donc la production de matériel en LN aux ateliers de Niono. À ma connaissance, ce matériel ne comprend aucune grammaire pédagogique. (Celles de l'équipe NUFU, bien que reconnues par le Ministère de l'Éducation en 2006, ne sont pas encore diffusées dans les écoles.) Pour l'aspect pédagogique, il faut croire que c'est l'enseignement en L1 qui est ainsi jugé positif, car l'approche globale de la lecture est remise en cause de manière générale et la base théorique de la $\mathrm{PC}$ critiquée notamment par $\mathrm{B}$. Maurer (2007). Les autres recommandations rappellent celles justifiant déjà le Curriculum (" capitaliser les acquis de la PC et corriger les faiblesses constatées de son application », M. L. Traoré 2009, cité en 2.1.3).

Parmi les points faibles figurent toujours l'absence d'un enseignement portant sur les LN dans la formation initiale des maîtres, ainsi que l'affectation des enseignants dans des régions dont ils ne maîtrisent pas la langue locale. On recommande ainsi de

\footnotetext{
transformer et améliorer les IFM [Institut de Formation des Maîtres] pour la formation des maîtres et des éducateurs du préscolaire [...] [pour] leur permettre de maîtriser les compétences professionnelles nécessaires à l'exercice de leur métier. À ce titre, il s'agit de
}

$[\ldots]$

introduire l'enseignement des langues nationales afin de pourvoir toutes les aires sociolinguistiques en enseignants locuteurs : il s'agira de tenir compte de la politique de promotion des langues nationales dans la formation initiale (Forum National 2008a : 46).

Le Forum souhaite aussi mieux intégrer les médersas dans le système éducatif, entre autres en élargissant le répertoire linguistique de leurs maîtres, qui en principe enseignent en langue arabe. Il faut notamment "multiplier les instituts de formation des maîtres des medersas et soutenir l'enseignement du français et des langues nationales dans ce sous-secteur » (Forum National 2008a : 47).

Quant à la formation linguistique en LN, elle semble toujours se limiter à la transcription, sans prendre en compte la structure des langues, car sous les recommandations pour les 
programmes scolaires, on trouve la précision suivante : "accorder un temps de formation suffisant pour la maîtrise des modules, notamment les innovations pédagogiques et les transcriptions des langues nationales » (Forum National 2008a : 49).

Les recommandations et précisions concernant les LN nationales n'apparaissent cependant de manière systématique qu'au chapitre « De l'éducation non formelle et de la politique des langues nationales » (op. cit. : 55-57). Malgré la position pionnière du Mali en matière d'éducation bilingue à l'école fondamentale, on lit ici :

\footnotetext{
L'enseignement des et en langues nationales est essentiellement associé à l'éducation non formelle, qui comprend les Centres d'Alphabétisation Fonctionnelle (CAF), les Centres d'Éducation pour le Développement (CED), et les Centres d'Apprentissage Féminins (CAFé). On recommande de prendre en compte aussi les CEI (Centres d'Éducation pour l'Intégration) (Forum National 2008a : 55).
}

La description des difficultés et les recommandations qui suivent vont pourtant au-delà du secteur informel. Parmi les défis se trouve "l'insuffisance de supports et matériels didactiques (productions scientifiques, dictionnaires, lexiques spécialisés etc. en langues nationales » (op. cit.: 55) - toujours sans mention du manque de grammaires pédagogiques... D'autres « insuffisances » touchant le système formel sont la formation et le nombre des enseignants ainsi que le suivi pédagogique des CAP et les coûts élevés de l'édition au Mali. Quant à "l'insuffisance d'environnement lettré en langues nationales ou un environnement lettré mal formulé » (ibid.), elle est commune aux secteurs formel et informel. Ces points faibles et les recommandations pour y remédier sont déjà identifiés par les travaux de recherches. Voici une sélection des recommandations, celles touchant à l'enseignement formel et aux conditions externes à l'école, nécessaires pour réussir dans ce secteur :

- $\quad$ utiliser les langues nationales dans tous les domaines de la vie publique pour assurer un véritable développement endogène du pays ;

- $\quad$ élaborer et utiliser des pièces d'état civil bilingue en langues nationales et français ;

- $\quad$ élaborer une politique linguistique cohérente partagée et acceptée de tous où les langues nationales sont considérées comme les premières langues de travail ;

- $\quad$ mettre en place un dispositif au niveau de l'enseignement Supérieur pour renforcer l'instrumentation des langues nationales ;

- renforcer la convergence des langues nationales et du Français [sic] dans le processus d'apprentissage jusqu'au lycée, dans les écoles publiques et privées ;

- former des professeurs de langues nationales à l'ENSUP [École Normale Supérieure]

- utiliser les langues nationales dans les divers ordres d'enseignement, y compris l'enseignement professionnel et l'enseignement supérieur (op. cit. : 55-56).

Il faut espérer que cette fois, les recommandations soient mieux suivies que par le passé et qu'il ne s'agisse pas de vœux pieux. Car, dans le document de synthèse, Déclaration $d u$ Forum national sur l'éducation, la question de la langue d'enseignement n'est pas mentionnée...

\section{Conclusion}

L'examen des documents officiels montre que le Mali invoque différents objectifs pour l'introduction des LN : dans la première phase (1979-1987), surtout la valorisation des langues, et dans les seconde et troisième phases (1987-2008), surtout le bilinguisme fonctionnel. Celui-ci s'inscrit dans un but plus large, le développement, tant de l'individu que du pays. On continue cependant à référer à la culture nationale, créant ainsi une certaine tension entre objectifs identitaire et instrumental: on ne sait s'il s'agit de promouvoir en premier lieu les LN ou le français. 
Les recherches en éducation bilingue permettent de constater qu'il y a aussi des contradictions entre les objectifs et les modèles choisis. Ainsi, pour la promotion des LN, la première expérimentation choisit non les modèles de maintien ou d'enrichissement mais le modèle de transition, qui vise l'intégration à la langue et culture dominante, le français.

$\mathrm{Si}$, en optant pour le bilinguisme fonctionnel, la $\mathrm{PC}$ et le Curriculum choisissent en apparence un modèle de maintien, les inégalités observées dans la distribution des deux langues à l'école, qui reflètent la situation diglossique de la société ambiante, font qu'en réalité, c'est toujours le modèle de transition qui prévaut. De plus, les points faibles de la méthode pédagogique de la PC (notamment l'approche structuro-globale de la lecture et l'absence d'un enseignement systématique de la grammaire des LN) jouent négativement sur les résultats scolaires.

Les conséquences de ces contradictions s'observent sur le terrain. Les enquêtes faites par l'équipe NUFU et les observations d'autres chercheurs permettent de constater que la réforme n'a atteint ni les buts linguistiques de maîtrise de L1 et L2 et de transition de l'une à l'autre, ni le but culturel de revalorisation des LN, ni le but social d'amélioration des résultats scolaires.

Les parents ne sont pas dupes, qui préfèrent le français comme langue d'instruction pour leurs enfants, malgré l'échec maintes fois constaté de l'école "classique». Pour qu'ils adhèrent à l'instruction en LN, il faudrait d'abord une politique linguistique où l'utilité des LN à l'écrit en dehors de l'école soit réelle, par exemple la possibilité pour les citoyens de s'adresser aux autorités et à l'administration dans la langue locale ou régionale, comme le recommande le Forum National sur l'Éducation de 2008.

Il faudrait ensuite des manuels de grammaire en LN, car la transcription seule ne saurait suffire. Aucun pays de longue tradition écrite ne se limite à cet aspect de l'écriture. D'ailleurs, dans ces langues, les livres de grammaire abondent, destinés non seulement à l'enseignement fondamental et normal, mais aussi aux niveaux secondaire et supérieur et à la recherche.

Le rôle des maîtres est primordial pour le succès des élèves en toute matière. La lecture et l'écriture étant à la base de tout apprentissage, leur formation initiale et continue en LN (grammaire et didactique de la L1) est indispensable pour la réussite de l'enseignement bilingue.

Enfin, il faut former les formateurs des formateurs. Que les Maliens soient encore obligés d'aller à l'étranger pour obtenir un diplôme supérieur dans leurs propres langues est d'autant plus paradoxal que le Mali prône depuis l'indépendance la promotion des ses LN. Le temps me semble venu de créer un enseignement supérieur des LN en tant que matière entière : une licence, un master et un doctorat, en bambara, fulfulde, songhay, etc., à l'université au Mali.

Si le Mali se donne ces moyens, on peut espérer atteindre aussi bien le but culturel que les buts linguistique et social de l'enseignement bilingue en français et en LN.

$\begin{array}{ll}\text { Abréviations } & \\ \text { APE } & \text { Association des Parents d'Élèves } \\ \text { AUF } & \text { Agence Universitaire de la Francophonie } \\ \text { CAF } & \text { Centre d'Alphabétisation Fonctionnelle } \\ \text { CAFé } & \text { Centre d'Apprentissage Féminin } \\ \text { CAP } & \text { Centre d'Animation Pédagogique } \\ \text { CED } & \text { Centre d'Éducation pour le Développement } \\ \text { CEI } & \text { Centre d'Éducation pour l'Intégration } \\ \text { CGS } & \text { Comité de Gestion Scolaire } \\ \text { CIAVER } & \text { Centre International Audio-Visuel d'Études et de Recherches, Sainth- } \\ & \text { Ghislain, Belgique }\end{array}$


CNRST Centre National de la Recherche Scientifique et Technologique

CPS Cellule de Planification et de Statistiques

DP Développement de la Personne (domaine d'enseignement à l'école fondamentale)

ENSUP École Normale Supérieure

FLASH Faculté de Lettres, Langues, Arts et Sciences Humaines, Université de Bamako

IFM Institut de Formation des Maîtres

INALCO Institut National des Langues et Civilisations Orientales, Paris

ISFRA

Institut Supérieur de Formation et de Recherche Appliquée

L1

Langue première

$\mathrm{L} 2$

Langue seconde

LC Langues et Communication (domaine d'enseignement à l'école fondamentale)

LL Langue, Littérature (filière au lycée)

LN Langue nationale

NEF Nouvelle École Fondamentale

NUFU Programme norvégien de recherche et de formation pour le développement (Universitets- og høyskolerådets program for utviklingsrelatert forskning og utdanning)

ONG Organisation Non Gouvernementale

PC Pédagogie Convergente

PRODEC Programme Décennal de Développement de l'Éducation

s.d. sans date

SGAV [Méthode] Structuro-Globale Audio-Visuelle

$\mathrm{SH}$

Sciences Humaines (domaine d'enseignement à l'école fondamentale)

SIL Summer Institute of Linguistics

SMT Sciences, Mathématiques et Technologie (domaine d'enseignement à l'école fondamentale)

s.p. $\quad$ sans pagination

TBS Taux Brut de Scolarisation

\section{Références}

AUF (Agence Universitaire de la Francophonie). s. d., s. p. Cours d'initiation à la didactique du Français Langue Étrangère en contexte syrien. www.lb.refer.org/fle/cours/cours3_AC/hist_didactique/cours3 hd09.htm (consulté le 14.05.10).

Baker, Victoria. 1998. Literacy in Developing Societies. Native language versus national language literacy. In : Aydin Y. Durgunoglu et Ludo Verhoeven. (eds.). Literacy Development in a Multilingual Context. Cross-cultural perspectives. Mahwah - New Jersey - London : Lawrence Erlbaum Associates, Publishers, 21-36. (accessible sur le site de Google Books).

Bamanankan maben nyebilagafe (ka nyesin karamogow ma). 2006. Y. Diallo, A. T. Doumbia, G. Dumestre, M. L. Haïdara, M. Konta, M. Niakaté, S. Simpara et S. Traoré. Bamako : Éditions Donniya. 
Banque mondiale. 2007. L'éducation au Mali. Diagnostic pour le renouvellement de la politique éducative en vue d'atteindre les objectifs du Millénaire. Washington D.C.

Bouwman, Dinie. 2005. Throwing Stones at the Moon : the role of Arabic in contemporary Mali. Ph.D. Thesis, Université de Leiden.

Brock-Utne, Birgit et Ingse Skattum (eds.). 2009. Languages and Education in Africa. A comparative and transdisciplinary analysis. Oxford : Symposium Books.

Calvet, Louis-Jean. 1987. La guerre des langues et les politiques linguistiques. Paris : Payot.

Calvet, Louis-Jean. 1988. Évaluation des classes expérimentales en bambara dans la région de Ségou. Bamako.

Calvet, Louis-Jean. 1992. Les langues des marchés au Mali. In : Louis-Jean Calvet. (éd.). Les langues des marchés en Afrique. Paris : Didier Érudition, 193-218.

Canvin, Maggie. 2003. Language and Education in Mali : A consideration of two approaches. Ph.D., University of Reading. SIL Publications. (Summer Institute of Linguistics).

Canvin, Maggie. 2007. Language and Education Issues in Policy and Practice in Mali, West Africa. In : Naz Rassool. (ed.). Global Issues in Language, Education and Development : Perspectives from post-colonial countries. Bristol : Multilingual Matters, 157-186. (accessible sur le site de Google Books).

Canut, Cécile et Gérard Dumestre. 1993. Français, bambara et langues nationales au Mali. In : Didier de Robillard et Michel Beniamino. (éds.). Le français dans l'espace francophone, Paris : Champion, tome 1, 219-228.

Chaudenson, Robert. 1989. 1989. Vers une révolution francophone?. Paris : L'Harmattan.

Chaudenson, Robert. 2006. Éducation et langues. Français, créoles, langues africaines. Paris, Organisation Internationale de la Francophonie, L'Harmattan.

CIAVER (Centre International Audio-Visuel d'Études et de Recherches). 1995. Rencontres. 1. Livre du maître. Paris : Agence de Coopération Culturelle et Technique.

Commission Nationale de Refondation du Système Éducatif Malien. 1995. Utilisation des langues nationales dans le système éducatif malien (Cas de l'Éducation de Base). Bamako, décembre.

Constitution. 1992. http://democratie.francophonie.org/IMG/pdf/Mali-2.pdf (consulté le 22.12.09).

Cummins, James. 1979. Linguistic Interdependence and the Educational Development of Bilingual Children. Review of Educational Research 49, 222-251.

Dembélé, Lamine. 2006. Conception des manuels scolaires. Bamako: Atelier de formation des formateurs d'enseignement des écoles à curricula.

Diarra, Abou et Youssouf M. Haïdara. 1999. Étude sociolinguistique sur l'identification des langues nationales dominantes par zone et du potentiel enseignant par langue. Ministère de l'Éducation de Base, Secrétariat Général. Octobre.

Direction Régionale de l'Éducation de Ségou. 1997. $4^{\text {ème }}$ stage de formation de haut niveau à la pédagogie convergente du 12 au 22 février 1997. Ségou.

Doumbia, Amadou Tamba. 2000. L'enseignement du bambara selon la pédagogie convergente au Mali : théorie et pratiques. Nordic Journal of African Studies 9-3 : 98-107. www.njas.helsinki.fi/

Dumestre, Gérard. 1994a. La dynamique des langues au Mali : le trinôme langues régionales bambara - français. In : Gérard Dumestre. (éd.). 1994b, 3-11. 
Dumestre, Gérard (éd.). 1994b. Stratégies communicatives au Mali : langues régionales, bambara, français. Paris : Didier Érudition.

Dumestre, Gérard. 1997. De l'école au Mali. Nordic Journal of African Studies 6- 2 :31-52.

Dumestre, Gérard. 2000. De la scolarité souffrante (Compléments à « De l'école au Mali »). Nordic Journal of African Studies 9-3 : 172-186. www.njas.helsinki.fi/

L'état de l'Afrique 2010. 2010. Paris : Jeune Afrique, hors série nº. 24.

Ethnologue: Languages of the World. 2005. $15^{\text {ème }}$ éd. (eds. R. G. Gordon et B. F. Grimes). Dallas, Texas : Summer Institute of Linguistics International.

Forum National sur l'Éducation. 2008a. Rapport général (30 et 31 octobre $-1^{\text {er }}$ et 2 novembre 2008). Bamako. www.education.gov.ml/IMG/pdf/Rapport_general_Forum_Final.pdf (consulté le 22.12.09).

Forum National sur l'Éducation. 2008b. Déclaration. Bamako. www.education.gov.ml/spip.php?article120 (consulté le 22.12.09).

Haïdara, Mamadou Lamine. 2000. Introduction des langues nationales dans l'enseignement : attitude des maitres de Bamako. Nordic Journal of African Studies 9-3 : 49-65. www.njas.helsinki.fi/

Haïdara, Mamadou Lamine. 2005. Problématique de l'enseignement des/en langue nationales : le cas du Mali. Thèse, Institut Supérieur de Formation et de Recherche Appliquée, Université de Bamako.

Haïdara, Youssouf M. 2003. Informations sur la Pédagogie Convergente. Bamako : Ministère de l'Éducation Nationale, Secrétariat général.

Hornberger, Nancy H. 1991. Extending Enrichment Bilingual Education : Revisiting typologies and redirecting policy. In : Ofelia Garcia. (ed.). Bilingual Education. Focus-schrift in honor of Joshua A. Fishman. Amsterdam - Philadelphia : John Benjamins, 215-234.

Hutchison, John, Abou Diarra et Joseph Poth. 1990. Évaluation de l'expérimentation en langues nationales dans l'enseignement fondamental en République du Mali. Washington : Agence des États-Unis pour le Développement International.

Kanè, Soumana. 2000. Manuels utilisés dans l'enseignement de la langue dans les écoles à pédagogie convergente : disponibilité et utilisation. Nordic Journal of African Studies 9-3 : 66-79. www.njas.helsinki.fi/

Kanouté, Mamadou Lamine. 2000. Mathématiques et langue nationale en milieu scolaire bambara. Nordic Journal of African Studies 9-3 : 80-97. www.njas.helsinki.fi/

Krinic et M. Wambach. 1997. Méthodologie de l'apprentissage de la langue nationale et des mathématiques. Saint-Ghislain, Belgique: CIAVER (Centre International AudioVisuel d'Études et de Recherches).

Loi $n^{\circ} 99$ / 046 / du 28 décembre 1999 portant loi d'orientation sur l'éducation. Bamako: Présidence de la République.

Maurer, Bruno. 2007. De la pédagogie convergente à la didactique intégrée: langues africaines - langue française. Paris : L'Harmattan.

Maurer, Bruno. 2010. Éléments de réflexion pour une didactique du plurilinguisme en Afrique francophone. Les Cahiers de l'Acedle 7- 1 : 167-179. http://acedle.org/IMG/pdf/Sommaire_Presentation_Cahiers-Acedle_7-1.pdf (consulté le 14.05.2010). 
Mbodj-Pouye, Aïssatou. 2007. Des cahiers au village. Socialisation à l'écrit et pratiques d'écriture dans la région cotonnière du sud du Mali. Thèse de doctorat de sociologie, Faculté de sociologie et d'anthropologie, Université Lumière-Lyon 2.

Mbodj-Pouye, Aïssatou et Cécile Van den Avenne. 2009. Comment les langues se mélangentelles à l'écrit ? Pratiques actuelles de deux agriculteurs passés par une école bilingue (franco-bambara) au Mali. In : Brock-Utne et Skattum. (eds.), 301-312.

Ministère de l'Éducation de Base, Institut Pédagogique National. 1994a. Programme du premier cycle de l'enseignement fondamental. Bamako.

Ministère de l'Éducation de Base. 1994b. Education de base au Mali. Problèmes et perspectives. Bamako. Octobre.

Naatirde naahu fulfulde. Eveil à la langue fulfulde. 2006. Traduit du bamanankan en fulfulde et adapté par Gouro Diall, Hamidou Sankaré et Samba Traoré. Bamako : Éditions Donniya.

Naparé, Hamidou. 2010. Langues nationales et politique d'éducation au Mali : analyse des contraintes sociolinguistiques et pédagogiques de l'enseignement en dogon. Thèse, Institut Supérieur de Formation et de Recherche Appliquée, Université de Bamako.

Nordic Journal of African Studies (Helsinki University Press), 9-3, numéro spécial : «L'école et les langues nationales au Mali ». (éd. Ingse Skattum). www.njas.helsinki.fi/

Opheim, Marianne. 1999. L'éducation bilingue au Mali. Le cas de Dougoukouna, « une école expérimentale en bambara ». Mémoire de maîtrise, Université d'Oslo.

Opheim, Marianne. 2000. Les filles et l'école au Mali. Nordic Journal of African Studies 9-3: 152-171. www.njas.helsinki.fi/

Plungian, Vladimir et Issiaka Tembiné. 1994. Vers une description sociolinguistique du pays dogon : attitudes linguistiques et problèmes de standardisation. In : Gérard Dumestre. (éd.). 1994b, 163-96.

PRODEC (Programme Décennal de Développement de l'Éducation). 2000. Les grandes orientations de la politique éducative. Bamako, janvier.

Sangaré, Coumba Touré. 2007. Problèmes didactiques et linguistiques de l'enseignement de la philosophie au Mali. Une étude de compréhension de textes par les lycéens de langue première bamanankan et fulfulde. Thèse, Institut Supérieur de Formation et de Recherche Appliquée, Université de Bamako.

Skattum, Ingse. 1997. L'éducation bilingue dans un contexte d'oralité et d'exoglossie : théories et réalités du terrain au Mali. Nordic Journal of African Studies 6-2 : 74-106. http://www.duo.uio.no/publ/IKOS/1997/74718/NJAS 1997_34.pdf

Skattum, Ingse. 2000. Le bambara écrit à l'école fondamentale. Nordic Journal of African Studies 9-3 : 103-127. www.njas.helsinki.fi/

Skattum, Ingse. 2004. L'apprentissage du français dans un pays « francophone » : le cas du Mali. In : Jan Jansen. (éd.). Mande - Manding. Background Reading for Ethnographic Research in the Region South of Bamako. Leyden : Department of Cultural Anthropology and Development Sociology, Leiden University, 105-117.ikos/personer/vit/ingse/L_apprentissage_du_francais_au_Mali.pdf

Skattum, Ingse. 2008. Mali : In Defence of Cultural and Linguistic Pluralism. In : Andrew Simpson. (éd.). Language \& National Identity in Africa. Oxford: Oxford University Press, 98-121. (Accessible sur le site de Google Books). 
Tamari, Tal. 2009. The Role of National Languages in Mali's Modernising Islamic Schools (Madrasa). In : Brock-Utne et Skattum. (eds.), 163-174.

Traoré, Mamadou Lamine. 2009. L'utilisation des langues nationales dans le système éducatif malien : historique, défis et perspectives. In : Brock-Utne et Skattum. (eds.), 155-161.

Traoré, Samba. 2000. La formation des maîtres du $1^{\mathrm{er}}$ cycle de l'enseignement fondamental au Mali : problèmes et perspectives. Nordic Journal of African Studies 9-3 : 29-48. www.njas.helsinki.fi/

Tréfault, Thierry. 1999. L'école malienne à l'heure du bilinguisme. Deux écoles rurales de la région de Ségou. Paris : Didier Érudition. 